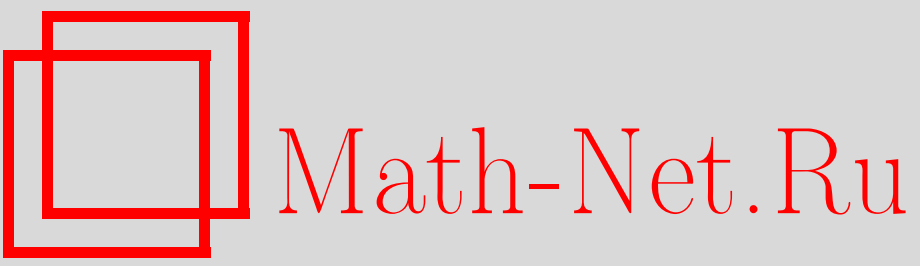

А. М. Райгородский, Задачи Борсука и Хадвигера и системы векторов с запретами на скалярные произведения, УМН, 2002, том 57, выпуск 3, 159-160

DOI: https://doi.org/10.4213/rm523

Использование Общероссийского математического портала Math-Net.Ru подразумевает, что вы прочитали и согласны с пользовательским соглашением

http://www.mathnet.ru/rus/agreement

Параметры загрузки:

IP: 54.162 .27 .143

26 апреля 2023 г., 16:25:24 


\section{ЗАДАЧИ БОРСУКА И ХАДВИГЕРА И СИСТЕМЫ ВЕКТОРОВ С ЗАПРЕТАМИ НА СКАЛЯРНЫЕ ПРОИЗВЕДЕНИЯ}

\section{А. М. РАЙГОРОДСКИЙ}

1. Введение и формулировка результата. Настоящая работа возникла в результате изучения двух задач комбинаторной геометрии - задачи Борсука и задачи Хадвигера. Первая задача состоит в нахождении минимального числа $f(d)$ такого, что всякое ограниченное множество $\Omega \subset \mathbb{R}^{d}$ может быть разбито на $f(d)$ частей меньшего диаметра (в [1] Борсук высказал гипотезу, что $f(d)=d+1)$. Вторая задача (см. [2]) связана с определением так называемого хроматического числа евклидова пространства, т.е. величины $\chi\left(\mathbb{R}^{d}\right)$, равной наименьшему числу красок, необходимых для такой раскраски всего $\mathbb{R}^{d}$, при которой расстояние между точками одного цвета $\neq 1$. В обеих задачах были получены многочисленные результаты. Так, например, гипотеза Борсука была доказана при $d \leqslant 3$, а также для произвольных выпуклых тел с гладкой границей. В то же время в достаточно болшших размерностях были найдены контрпримеры: доказано, что гипотеза неверна, по крайней мере, при всех $d \geqslant 323$ (см. [3] и [4]) и что имеет место оценка $f(d) \geqslant(1.2255 \ldots+o(1))^{\sqrt{d}}($ см. [5]). В задаче Хадвигера наилучшими из известных являются, в частности, неравенства $4 \leqslant \chi\left(\mathbb{R}^{2}\right) \leqslant 7$ и $(1.239 \ldots+o(1))^{d} \leqslant \chi\left(\mathbb{R}^{d}\right) \leqslant(3+o(1))^{d}$ (см. [6]-[9]). Наконец, сравнительно недавно выяснилось, что некоторьй общий подход, связанный с рассмотрением решетчатых многогранников, позволяет получать нетривиальные нижние оценки как для $f(d)$, так и для $\chi\left(\mathbb{R}^{d}\right)$. Описание этого подхода, а также более подробную историю изложенных вопросов можно найти в [10]. Здесь же мы заметим лишь, что, в свою очередь, в рамках упомянутого подхода наиболее эффективно используется техника, относящаяся к экстремальной теории гиперграффов с запретами на мощности пересечения ребер и восходящая к работе Франкла и Вилсона (см. [11]). Такая техника была в существенной степени доработана и обобщена автором, которому удалось показать важность изучения экстремальных свойств систем $(0,1,-1)$-векторов (см. замечание 1 в следующем пункте). В настоящей заметке будет изложен новый метод получения верхних оценок для мощностей некоторых таких систем с запретами на скалярные произведения.

Пусть фиксированы натуральные числа $n, k, b=2^{k}$ с условием, что $\frac{n}{2} \leqslant b \leqslant c n, \frac{1}{2}<c<1$. Пусть, кроме того, $\mu$ есть число нечетных чисел на отрезке $[2 b-n, b]$, а $\eta$ есть число четных чисел на отрезке $[2, b-2]$. Рассмотрим систему векторов $\Sigma=\left\{\mathbf{x}=\left(x_{1}, \ldots, x_{n}\right) \in\{0,1,-1\}^{n}\right.$ : $\left.\left|\left\{x_{i}= \pm 1\right\}\right|=b\right\}$ и предположим, что первая ненулевая координата в каждом из векторов равна единице. Тогда имеет место

Теорема. Какова бы ни была подсистема векторов $Q=\left\{\mathbf{x}_{1}, \ldots, \mathbf{x}_{s}\right\} \subset \Sigma$, удовлетворяющая условиям $\left(\mathbf{x}_{i}, \mathbf{x}_{j}\right) \neq 0 \quad \forall i \neq j$, выполнена оценка $s \leqslant P(n) \max _{t} C_{n}^{\mu+t} C_{n-\mu-t}^{\eta-2 t}$, где максимум берется по всем натуральным $t \in[0, \eta / 2]$, а $P(n)$ есть некоторый полином.

2. Доказательство теоремы и некоторые замечания. Предлагаемое доказательство опирается на методы линейной алгебры в комбинаторике. Для каждой пары векторов $\mathbf{x}, \mathbf{y} \in \Sigma$ мы определим величину $|\mathbf{x} \cap \mathbf{y}|=\sum_{i=1}^{n} x_{i}^{2} y_{i}^{2}$, которая равна мощности пересечения множеств ненулевых координат этих векторов. Заметим сразу, что $|\mathbf{x} \cap \mathbf{y}| \equiv 0(\bmod 2)$ тогда и только тогда, когда $(\mathbf{x}, \mathbf{y}) \equiv 0(\bmod 2)$. Положим $\nu=\sum_{i=1}^{\infty}\left[(b-2) / 2^{i}\right]$ и каждому вектору $\mathbf{x} \in \Sigma$ сопоставим полином $F_{\mathbf{x}} \in \mathbb{Q}\left[y_{1}, \ldots, y_{n}\right]: F_{\mathbf{x}}(\mathbf{y})=2^{-\nu} \prod_{l}(l-|\mathbf{x} \cap \mathbf{y}|) \times \prod_{m}(m-(\mathbf{x}, \mathbf{y}))$, где $l$ пробегает множество всех нечетных чисел на отрезке $[2 b-n, b]$ (т.е. количество сомножителей в первом произведении равно $\mu$ ), $m$ четно и меняется в пределах от двойки до $b-2$ (так что степень второго произведения равна $\eta$ ), а выражение $|\mathbf{x} \cap \mathbf{y}|$ можно, вообще говоря, понимать, отвлекаясь от его изначального определения, и как формальную запись $\sum_{i=1}^{n} x_{i}^{2} y_{i}^{2}$, имеющую смысл

Работа выполнена при финансовой поддержке Российского фонда фундаментальных исследований (гранты № 02-01-00912, 00-15-96109). 
не только для $\mathbf{y} \in \Sigma$. Теперь от полиномов $F_{\mathbf{x}}$ мы стандартньм образом перейдем к полиномам $\widetilde{F}_{\mathbf{x}} \in \mathbb{Q}\left[y_{1}, \ldots, y_{n}\right]$, последовательно применяя соотношения $y_{i}^{3}=y_{i}, i=1, \ldots, n$, которые, во всяком случае, верны для координат векторов из системы $\Sigma$ (ср., скажем, [5]). Нетрудно убедиться в том, что для построенных полиномов и пар $\mathbf{x}, \mathbf{y} \in \Sigma$ вьполнены следующие свойства: а) если $(\mathbf{x}, \mathbf{y})$ нечетно, то $\widetilde{F}_{\mathbf{x}}(\mathbf{y})=0 ;$ б) если $(\mathbf{x}, \mathbf{y})$ четно и не равно ни нулю, ни $b$, то $\widetilde{F}_{\mathbf{x}}(\mathbf{y}) \equiv 0(\bmod 2) ;$ в) если $(\mathbf{x}, \mathbf{y}) \in\{0, b\}$, то $\widetilde{F}_{\mathbf{x}}(\mathbf{y}) \equiv 1(\bmod 2)$. (Здесь мы фактически пользуемся замеченньм выше свойством одновременной четности величин $(\mathbf{x}, \mathbf{y})$ и $|\mathbf{x} \cap \mathbf{y}|$ и определением числа $\nu ;$ кроме того, ясно, что всегда $|\mathbf{x} \cap \mathbf{y}| \in[2 b-n, b]$.) Рассуждения, аналогичные тем, что были впервые проведены автором в [3] и более деталшно изложены им в [10], показывают, что из свойств а)-в) следует линейная независимость над $\mathbb{Q}$ полиномов $\widetilde{F}_{\mathbf{x}_{1}}, \ldots, \widetilde{F}_{\mathbf{x}_{s}}$, где векторы $\mathbf{x}_{1}, \ldots, \mathbf{x}_{s}$ суть элементы произволшной подсистемы $Q \subset \Sigma$ из формулировки теоремы. Стало быть, $s$ не превосходит размерности пространства, порожденного полиномами $\widetilde{F}_{\mathbf{x}}, \mathbf{x} \in \Sigma$. В свою очередь, из конструкции полиномов немедленно вытекает искомое неравенство: $s \leqslant P(n) \max _{t} C_{n}^{\mu+t} C_{n-\mu-t}^{\eta-2 t}$.

ЗАмечАние 1. Доказанная теорема напрямую связана с гипотезой автора (см. [10]) о том, что при $b=n / 2$ правилшной является оценка $s \leqslant 2^{n}$. Тем самшм, с одной стороны, нами предложен новый подход к доказательству этой гипотезы и к получению нетривиальных смежных результатов. С другой стороны, нами найдено некоторое обоснование того факта, что в гипотезе фигурирует именно степень двойки $\left(n=2^{k+1}\right)$ : в самом деле, деталшный анализ нашего метода показьвает, что в его рамках (при доказательстве линейной независимости полиномов) работать нужно с классами вьчетов по четному модулю и что, более того, модуль обязан иметь вид $p^{\alpha}$. Отметим, что из гипотезы вытекает возможность опровержения гипотезы Борсука при всех $d \geqslant 135$ и получения оценок $f(d) \geqslant(1.632 \ldots+o(1))^{\sqrt{d}}$ и $\chi\left(\mathbb{R}^{d}\right) \geqslant(1.414 \ldots+o(1))^{d}$.

ЗАмечАниЕ 2. Резулштаты, аналогичные доказанным, могут быть также сформулированы и для случаев, когда в каждом векторе рассматриваемой системы $\Sigma$ фиксировано число отрицателшных координат. Такого рода результаты позволяют улучшать константу в нижней оценке для $\chi\left(\mathbb{R}^{d}\right)$, но в настоящей работе мы на них не останавливаемся.

\section{СПИСОК ЛИТЕРАТУРЫ}

[1] K. Borsuk // Fund. Math. 1933. V. 20. P. 177-190. [2] H. Hadwiger // Portugal. Math. 1944. V. 4. Р. 140-144. [3] А. М. Райгородский // УМН. 1997. Т. 52. № 6. С. 181-182. [4] A. Hinrichs // Discrete Math. 2002. V. 243. P. 253-256. [5] А. М. Райгородский // УMH. 1999. T. 54. № 2. C. 185-186. [6] L. Moser, W. Moser // Canad. Math. Bull. 1961. V. 4. P. 187-189. [7] Н. Hadwiger // Elem. Math. 1961. V. 16. P. 103-104. [8] А. М. Райгородский // УМH. 2000. T. 55. № 2. C. 147-148. [9] D. G. Larman, C. A. Rogers // Mathematika. 1972. V. 19. Р. 1-24. [10] А. М. Райгородский // УМН. 2001. Т. 56. № 1. С. 107-146. [11] P. Frankl, R. Wilson // Combinatorica. 1981. V. 1. P. 357-368.

Московский государственньй

Принято редколлегией университет им. М. В. Ломоносова 